\title{
REVIEW
}

\section{The Role of Topical Brimonidine Tartrate Gel as a Novel Therapeutic Option for Persistent Facial Erythema Associated with Rosacea}

\author{
Andrew William Johnson · Sandra Marchese Johnson
}

To view enhanced content go to www.dermtherapy-open.com

Received: May 20, 2015 / Published online: June 26, 2015

(C) The Author(s) 2015. This article is published with open access at Springerlink.com

\begin{abstract}
Rosacea is a chronic inflammatory skin condition that commonly presents with persistent facial erythema with or without the coincident presence of flushing, telangiectasias, inflammatory papules or pustules, phymatous changes, or ocular involvement. Patients often present with a constellation of various signs and symptoms of the disease, and an individualized treatment plan should be tailored to a patient's unique clinical presentation. Previously available medications for rosacea have all targeted the inflammatory erythematous papules and pustules frequently associated with the disease, leaving a therapeutic gap for the common manifestation of persistent facial erythema. Brimonidine tartrate $0.33 \%$ gel was
\end{abstract}

Electronic supplementary material The online version of this article (doi:10.1007/s13555-015-0078-1) contains supplementary material, which is available to authorized users.

A. W. Johnson

Department of Dermatology, University of Arkansas for Medical Sciences, Little Rock, AR, USA

S. M. Johnson ( $\square)$

Johnson Dermatology, Fort Smith, AR, USA

e-mail: drsandy@johnsondermatology.com approved by the US Food and Drug Administration in August 2013 as the first medication available for the topical treatment of persistent facial erythema associated with rosacea. Brimonidine gel is a highly selective $\alpha 2$ adrenergic receptor agonist with potent vasoconstrictive effects, which leads to significant reduction of persistent facial erythema in the majority of patients when applied once daily. Based on large-scale clinical trials and post-marketing reports, brimonidine gel has maintained a good safety profile with a minority of patients experiencing adverse effects from its use, most of which are cutaneous in nature, mild-to-moderate in degree, occur early after initiation of treatment, often resolve spontaneously with continued use, and generally resolve after discontinuation of use. Among the reported adverse effects, two distinct manifestations of worsened erythema have been described. Brimonidine gel can be integrated into a treatment regimen along with concomitant therapies for facial papules and pustules with no increased risk of adverse events with combination therapy. Education about optimal application methods, setting 
reasonable expectations for treatment, and minimizing inflammation are important factors for the successful use of brimonidine gel as part of a patient's overall rosacea treatment regimen.

Keywords: Brimonidine; Erythema; Flushing; Gel; Mirvaso; Redness; Rosacea; Topical; Treatment

\section{INTRODUCTION}

Rosacea is a chronic condition of the facial skin that affects approximately 16 million people in the United States [1]. Clinically, it is characterized by flushing, persistent facial erythema, telangiectasias, papules, pustules, edema, and/or phymatous changes. It can affect the facial skin as well as the eyes. Most experts currently view rosacea as a chronic inflammatory skin disease with a spectrum of clinical features that can wax and wane over time and that often overlap several of the different clinical subtypes [1-3]. According to the National Rosacea Society Expert Committee in 2002, rosacea is grouped into the four following clinical subtypes: erythematotelangiectatic (ETR), papulopustular (PPR), phymatous, and ocular [4]. ETR characteristically presents with flushing and persistent facial erythema, while PPR usually presents with inflamed papules and pustules. In the phymatous subtype, there is thickening of the skin as well as nodule formation causing irregularity of the surface of the facial skin. Ocular rosacea typically involves irritation or a burning sensation associated with redness of the eyes and is considered a clinical entity that is distinct from the three facial rosacea subtypes; however, it can be present in about $20 \%$ of those patients [4].
Although the four subtypes are characterized by different common signs and symptoms, patients often present with a range of symptoms that overlap several subtypes; and, transient and persistent facial erythema is often observed in all four subtypes of rosacea [5]. For the purposes of this review, ETR will be the subtype most frequently discussed. Facial redness is an extremely common manifestation of rosacea and presents a significant challenge for treatment. Vascular and inflammatory mechanisms are both involved in the clinical manifestations of this problem [6, 7]. PPR exhibits lesional or perilesional erythema that is distinct from generalized vascular erythema [8-10]. It is believed that the vascular mechanisms contributing to facial erythema in rosacea can be transient or persistent [8]. Flushing associated with rosacea likely occurs via a different mechanism than that of generalized erythema; but, flushing should also be considered distinct from physiologic blushing, which occurs involuntarily in response to emotional stimuli.

There is no cure for rosacea, and treatments are intended to control the signs and symptoms that define the disease [11]. Until recently, there have been only five medications approved by the US Food and Drug Administration (FDA) for the treatment of rosacea, and all of these are intended to treat the inflammatory lesions of the PPR subtype [12]. Metronidazole is a synthetic antimicrobial, antioxidant, and antiinflammatory agent recommended by the American Acne and rosacea Society (AARS) for the topical treatment of inflammatory lesions and transient erythema $[12,13]$. Azelaic acid is a naturally occurring saturated dicarboxylic acid with anti-inflammatory, antioxidant, and antimicrobial effects recommended by the AARS for the topical treatment of 
inflammatory papules and pustules of mild to moderate rosacea [12, 14]. Sulfur has keratolytic, antifungal, and antibacterial properties, and sodium sulfacetamide-sulfur is used for the topical treatment of inflammatory papular lesions in rosacea $[15,16]$. Ivermectin is a broad-spectrum anti-parasitic medication also with anti-inflammatory effects that is thought to kill Demodex mites residing in the pilosebaceous units of patients with PPR and has recently been approved for topical use [17]. Lastly, a sub-antimicrobial dose of the oral medication doxycycline $40 \mathrm{mg}$ modified release once daily is approved for the treatment of inflammatory lesions of rosacea in patients at least 18 years of age [12]. All of these approved medications target the inflammatory lesions (papules and pustules) of rosacea. Because of this there has been a gap in treatment options available for the persistent facial erythema of rosacea that is most pronounced in ETR but observed in all four subtypes of the disease.

The US FDA approved brimonidine tartrate 0.33\% gel in August 2013 for the topical treatment of persistent (nontransient) facial erythema of rosacea in adults 18 years of age and older. This is the first and currently only approved topical treatment for the persistent erythema of rosacea [18]. Brimonidine is a highly selective $\alpha 2$-adrenergic receptor agonist with potent vasoconstrictive effects [19]. Another $\alpha$-adrenergic receptor agonist, oxymetazoline (a selective $\alpha 1$ agonist and partial a2 agonist), is currently under investigation in phase 3 clinical trials for the topical treatment of facial erythema of rosacea [20]. This review article will discuss the mechanism of action of topical brimonidine gel in the treatment of persistent facial erythema in rosacea, the clinical data leading to its FDA approval for this indication, as well as its approved dose, administration, safety profile, reported adverse effects, and role in combination treatment for rosacea.

This article is based on previously conducted studies and does not involve any new studies of human or animal subjects performed by any of the authors.

\section{MECHANISM OF ACTION AND PHARMACOKINETICS}

Brimonidine is a highly selective $\alpha 2$-adrenergic receptor agonist and is 1000 -fold more selective for the $\alpha 2$-adrenergic receptor than the $\alpha 1$ adrenergic receptor [21]. This medication was previously used as a topical treatment for openangle glaucoma and was more recently found to be effective in controlling the diffuse facial erythema of rosacea. A recent study by Piwnica et al. demonstrated high selectivity of brimonidine specifically for the $\alpha 2-\mathrm{A}$ adrenergic receptor with minimal off-target effects from interactions with the $\alpha 1-A, \alpha 1-B$, $\alpha 2-B$, and $\alpha 2-C$ adrenergic receptors [21]. Through this specific action on post-synaptic endothelial $\alpha 2$ receptors, brimonidine was shown to be a potent vasoconstrictor of human subcutaneous blood vessels with a diameter of less than $200 \mu \mathrm{m}$, and it was also demonstrated to block the vasodilatory effect of capsaicin [21]. Additionally, the antiinflammatory nature of brimonidine in skin has been demonstrated in mouse ear models of inflammation, in which it was found to inhibit experimentally induced ear edema compared to a matched vehicle control [21].

Brimonidine is metabolized extensively by the liver, and the major route of its elimination and that of its metabolites is urination excretion [4]. In a recent study by Benkali et al., detectable systemic exposure occurred in $22-79 \%$ of 
patients who received topical brimonidine gel daily for 29 days with increasing concentrations from $0.18-0.5 \%$, respectively, and with average peak serum concentrations ranging from 13 to $25 \mathrm{pg} / \mathrm{mL}$ [22]. This very low level of systemic absorption was lower than that of brimonidine tartrate $0.2 \%$ ophthalmic solution three times daily, which yielded a mean peak serum concentration of $54 \mathrm{pg} / \mathrm{mL}$.

\section{PHASE II CLINICAL TRIAL DATA}

The pharmacodynamics and safety of three concentrations $(0.07,0.18$, and $0.5 \%)$ of topical brimonidine gel were evaluated in a first phase II randomized, double-blind, parallel-group, vehicle-controlled study (study A) [23]. This first study enrolled 122 patients (30 men, 92 women) and was conducted at 5 centers in the United States. The clinician's erythema assessment (CEA) and patient's selfassessment (PSA) scales were used to grade improvements in facial erythema at specified time points (Table 1). Brimonidine was effective in reducing erythema for the first $12 \mathrm{~h}$ in a dosedependent manner, with the greatest effect seen at a concentration of $0.5 \%$ [23]. All three concentrations were considered safe and were well tolerated.

A second phase II study (study B, or phase IIb) enrolled 269 patients (52 men, 217 women) at 17 centers in the United States and evaluated topical brimonidine gel at concentrations of $0.18 \%$ and $0.5 \%$ [23]. Once-daily topical administration demonstrated rapid onset, sustained duration of action, and a dosedependent effect with maximum benefit at $0.5 \%$ with no significant difference in the number of patients experiencing a perceived adverse effect compared to that in the vehicle control group [23]. During the 4 weeks of the study and the 4-week follow-up period after the study, there was no tachyphylaxis (loss of previous noted effect) or rebound (worsening of the persistent facial erythema compared to baseline) observed. The aim of these two studies was to determine the optimal dose regimen for the treatment of moderate to severe erythema associated with rosacea, and based on the results the concentration of $0.5 \%$ brimonidine gel was selected for phase III evaluation [23].

\section{PHASE III CLINICAL TRIAL DATA}

The safety and efficacy of brimonidine gel in the treatment of rosacea were evaluated in two large-scale, multicenter, randomized, doubleblind, parallel-group, vehicle-controlled phase III studies as well as a long-term open-label safety study [19, 24]. The first phase III study enrolled 260 patients (54 men, 206 women) in 15 centers in the US and in Canada. Patients with moderate to severe erythema due to rosacea were randomized to receive topical

Table 1 Clinician's erythema assessment (CEA) and patient's self-assessment (PSA)

\begin{tabular}{lll}
\hline Scores & CEA & PSA \\
\hline 0, Clear & Clear skin with no signs of erythema & Clear of unwanted redness \\
1, Almost clear & Almost clear; slight redness & Nearly clear of unwanted redness \\
2, Mild & Mild erythema; definite redness & Somewhat more redness than I prefer \\
3, Moderate & Moderate erythema; marked redness & More redness than I prefer \\
4, Severe & Severe erythema; fiery redness & Completely unacceptable redness \\
\hline
\end{tabular}


brimonidine $0.5 \%$ gel or a vehicle control once daily for 4 weeks with a 4-week follow-up period. A second phase III study enrolled 293 patients (80 men, 213 women) with the same study design and the same concentration of brimonidine $0.5 \%$ compared to vehicle control [19].

As in the phase II studies, the CEA and PSA scales were used to grade improvements in facial erythema at specified time points in the studies. The primary endpoint for both phase III studies was defined as a 2-grade improvement in both the CEA and PSA at 3-h intervals over the first 12 -h post-treatment on days 1,15 , and 29 , with a 1-grade improvement considered a clinically meaningful result. The secondary endpoint was defined as a 1-grade improvement from baseline in both the CEA and PSA at 30 min after application on day 1, which corresponded to the onset of action. Compared to the vehicle control, once-daily topical brimonidine $0.5 \%$ gel demonstrated a significant reduction in facial erythema based on both the CEA and the PSA 30 min after application on day 1 , and this significant effect was again demonstrated $30 \mathrm{~min}$ after application on days 15 and 29 [19]. On day 29 , significantly more patients had a 2-grade improvement on both the CEA and PSA at 3, 6, 9 , and $12 \mathrm{~h}$ after application of brimonidine $0.5 \%$ gel compared to vehicle control [19].

Once-daily brimonidine $0.5 \%$ gel was considered safe and well tolerated in both phase III studies with the incidence of adverse events (AEs) being 29.5\% compared to $25.2 \%$ for the vehicle control in the first study and $33.8 \%$ compared to $24.1 \%$ for the vehicle control in the second study [19]. The incidence of AEs related to brimonidine was $11.6 \%$ compared to $5.3 \%$ for the vehicle control in the first study and 9.5\% compared to $9.7 \%$ for the vehicle control in the second study [19].
As seen in the phase IIb study as well as during the 4-week study period and the 4-week followup period for both phase III studies, there was no tachyphylaxis or rebound erythema (as it was defined in the clinical trials) observed for the use of topical brimonidine gel, with the exception of isolated cases of worsening erythema and/or flushing [19]. These isolated cases were not recorded as "rebound."

\section{LONG-TERM SAFETY STUDY}

The long-term safety and efficacy of brimonidine $0.5 \%$ once daily were evaluated in a 12-month, open-label, multicenter study [24]. There were 449 patients enrolled in the study (113 men, 336 women), of which 335 (74.6\%) completed at least 6 months of oncedaily treatment and $279(62.1 \%)$ completed 12 months of treatment with follow-up (a total of 345 subject-years of exposure) [24]. The study included 8 visits: screening, baseline at day 1 , follow-up at week 1, and repeat follow-up at months 1, 3, 6, 9, and 12. Safety tests and measures included vital signs, intraocular pressure, physical exams, blood chemistry, hematology, and urinalysis. Treatment measures included the CEA, PSA, the 5-point Telangiectasia Grading Assessment scale from 0 (clear) to 4 (severe), and manual count of inflammatory lesions present (sum of papules, pustules, and nodules). AEs were also reported and assessed throughout the 12-month study.

AEs were most commonly reported during the first quarter $(41.9 \%$ total patients with AEs; $21.4 \%$ with AEs related to brimonidine use), with a substantial decline by the second quarter (24.6\% total AEs; $7.6 \%$ related AEs), followed by a relative decline for the remainder of the study (19.5\% total AEs in the fourth quarter; $4.2 \%$ related AEs in the fourth quarter) [24]. The most 
common related AEs observed were flushing (9.1\%), worsening of erythema (6.5\%), worsening of rosacea (3.6\%), contact dermatitis (2.2\%), and pruritus (2.0\%) [24]. The majority of these were mild or moderate in intensity. Approximately $30 \%$ of patients received concomitant therapies for their facial papules and pustules related to rosacea, and there was no observed increased risk for AEs from combination therapy with topical brimonidine treatment [24].

In terms of efficacy, the 12-month study corroborated the results of the phase II and phase III trials with a durable beneficial effect in the treatment of moderate to severe facial erythema associated with rosacea without any observed tachyphylaxis [24]. Long-term followup studies are needed to examine whether there is chronic remodeling of vasculature or durable modification of neurovascular regulation with prolonged daily use that may alter baseline persistent facial erythema associated with rosacea.

\section{DOSE, ADMINISTRATION, PRECAUTIONS, AND ADVERSE REACTIONS}

Brimonidine gel is intended to be applied as a pea-sized amount once daily to each of five regions of the face: the central forehead, chin, nose, and each cheek, with even application as a thin layer avoiding the eyes and lips. Each gram of gel contains $5 \mathrm{mg}$ of brimonidine tartrate, equivalent to $3.3 \mathrm{mg}$ of brimonidine-free base.

According to the FDA, brimonidine tartrate topical gel should be used with caution in patients with depression, cerebral or coronary artery insufficiency, Raynaud's phenomenon, orthostatic hypotension, thrombangiitis obliterans, scleroderma, or Sjögren's syndrome due to concern for possible potentiation of vascular insufficiency in these patients. It should also be used with caution in patients with severe or unstable cardiovascular disease, as a2-adrenergic agonists can lower blood pressure. These are theoretical risks to consider, and the clinical trials mentioned above demonstrate a high safety profile for this medication. It is, however, important to keep brimonidine gel out of reach of children as two young children of a research subject in one of the clinical trials experienced serious adverse reactions following accidental ingestion. This included lethargy, respiratory distress with apneic episodes requiring intubation, sinus bradycardia, confusion, psychomotor hyperactivity, and diaphoresis. Both children made a full recovery within $24 \mathrm{~h}$ with no sequelae [32].

The most common AEs were cutaneous and mild or moderate in degree. They include flushing $(9.1 \%)$, worsening erythema (6.5\%), worsening of papules and pustules of rosacea (3.6\%), burning sensation of the skin (3.3\%), skin irritation (3.1\%), contact dermatitis (2.2\%), and pruritus (2.0\%) [24]. Allergic contact dermatitis was reported in approximately $1 \%$ of patients, and results from patch testing of two clinical trial subjects revealed that one was sensitive to brimonidine tartrate and the other was sensitive to phenoxyethanol (preservative) $[32,33]$. Potential medication interactions include antihypertensives, cardiac glycosides, central nervous system depressants, and monoamine oxidase inhibitors. Topical brimonidine tartrate gel is pregnancy category $B$, has unknown excretion in human breast milk but has been shown to be excreted in breast milk in animal studies, and is not intended for use in children and should be kept out of their reach [32]. 


\section{PARADOXICAL ERYTHEMA AND EXAGGERATED RECURRENCE OF ERYTHEMA}

There have been recent case reports of exaggerated recurrence of erythema (documented in the literature as "rebound") associated with the use of topical brimonidine tartrate gel [25, 26]. One case described a 29-year-old female with ETR associated with persistent erythema and occasional flushing who had a favorable reduction in baseline erythema within 1-2 $\mathrm{h}$ after application that lasted for $10-12 \mathrm{~h}$ but was followed by an exaggerated recurrence of erythema past baseline, which lasted for 12-14 $\mathrm{h}$ and was self-limited [25]. This occurred again in the same patient with the two subsequent applications, and she then stopped using the medication. Another report outlined three patient cases in which persistent erythema was favorably reduced within 1-6 h after application followed by an exaggerated recurrence of erythema past baseline at $12 \mathrm{~h}$ after application that was associated with a burning sensation [26]. This lasted an additional $12 \mathrm{~h}$ and was self-limited with spontaneous resolution within 1 day, but it was reported to recur and worsen with continued use in these individuals. The cases reported by Routt et al. also involved worsening of erythema after the first application of brimonidine gel [26]. This relatively immediate reaction (with or without additional symptoms such as burning) was previously suggested to be considered a paradoxical erythema, which was defined as redness appearing within 3-6 $\mathrm{h}$ after application of brimonidine [27]. This should be considered as a different reaction than the exaggerated recurrence of erythema, and it can be more bothersome for patients since the erythema occurs during the time period when he or she most wanted to be free of facial redness.

Similar cases as those mentioned in the reports above have been reported to the manufacturer of brimonidine gel, Galderma Laboratories, L.P., as reports of "condition aggravated" or "rebound effect" as part of postmarketing pharmacovigilance analysis. In these reports from drug launch in August 2013 through April 2014, the most frequently associated symptoms were erythema, flushing, burning sensation or skin warmth, and rarely, skin pain [27]. Dermatitis, pruritus, swelling, and pallor were reported in less than $10 \%$ of these cases [27]. These reactions were reported to occur most often in the first 1 week of initiation of therapy [27]. There is usually rapid resolution of these AEs with only rare reports of paradoxical erythema or exaggerated recurrence of erythema with the aforementioned associated symptoms lasting weeks, and in a majority of cases the AE improved or resolved after stopping brimonidine [25-27].

Similar to the trend in the post-marketing pharmacovigilance data where it was observed that paradoxical erythema and exaggerated recurrence of erythema decreased dramatically after 1 week of use, erythema and a burning sensation were infrequent AEs of topical brimonidine use during the clinical trials; and, the incidence of all AEs decreased from quarter one through quarter four over the first 12 months of daily use [24].

\section{EXPERIENCES IN CLINICAL PRACTICE}

As an ophthalmic solution, brimonidine tartrate has a well-established safety and efficacy record with nearly 20 years of clinical use for the treatment of open-angle glaucoma 
$[28,29]$. After recent clinical trials have demonstrated its safety and efficacy in a gel formulation for the treatment of persistent facial erythema associated with rosacea, the FDA approved brimonidine tartrate $0.33 \%$ topical gel in August 2013. Clinical trial data, long-term pharmacovigilance data, and individual case reports in the literature describe infrequent adverse effects associated with use of brimonidine gel, most of which are cutaneous, mild, and reversible upon discontinuation of use, and among which are the occurrence of paradoxical erythema and exaggerated recurrence of erythema.

Tanghetti et al. recently published panel recommendations in 2015 regarding the optimal use of brimonidine gel in the management of rosacea [27]. First among these recommendations, one should assess the clinical features of rosacea and rule out alternative diagnoses followed by the development of a treatment plan that targets the different clinical symptoms present in each individual patient $[27,30]$. Second, each patient should be educated about triggers such as UV light, heat, spicy foods, red wine, among others; and, it should be clearly explained that brimonidine will not completely negate the erythema-inducing effects of these triggers, nor will it eliminate papules, pustules, or telangiectasias associated with rosacea or provide a cure for facial erythema associated with rosacea $[19,24,27,30]$. Steps should be taken to minimize inflammation associated with rosacea, including the use of gentle cleansers, moisturizers or skin barrier repair products, and regular photoprotection [27, 31]. Inflammatory lesions associated with rosacea should be treated with an appropriate agent when they are present because these, as well as perilesional erythema and telangiectasias, may be more visible following the use of brimonidine $[27,30]$. Appropriate expectations should be set, including discussion that there is a risk of worsening of facial redness in $10-20 \%$ of patients, that this worsening of redness usually occurs within the first 2 weeks of treatment when it does occur, and that this adverse effect generally resolves spontaneously within $12-24 \mathrm{~h}$ after discontinuation of use [27]. Tanghetti et al. suggested the potential use of oral acetyl salicylic acid $80-500 \mathrm{mg} /$ day, ibuprofen, or naproxen for burning sensations associated with worsening of facial redness, an antihistamine if swelling or pruritus is present, a cool compress for facial warmth, or a topical corticosteroid or calcineurin inhibitor for any of these associated symptoms [27]. Allergic or irritant contact dermatitis should be suspected, despite its rare occurrence, if facial redness first appears greater than 3-4 months after initiation of therapy [27]. Lastly, it is important to educate patients about optimal application of brimonidine: start with a small pea-sized amount spread thinly once every day, in some cases on a test patch prior to application of the entire face, with gradual increases in the amount applied to achieve the desired effect [27]. Patients should continue use of their facial moisturizer along with the initiation of brimonidine gel, and the first application should be on a day when the patient can stay home to observe the effects [27]. It is important to explain that since brimonidine gel has been studied and approved based on once-daily use, it is difficult to predict its side effect profile when used intermittently [27].

One of the authors of this paper (SMJ) has successfully integrated the use of brimonidine gel for the treatment of persistent facial erythema of rosacea by reinforcing good skin hygiene with all patients suffering from rosacea. This includes using only gentle cleansers, using only the fingers to cleanse the face, and 
avoiding the use of toners, astringents, exfoliants, washcloths, or other abrasive products. Liberal use of bland moisturizers and sun protection is also encouraged. When initiating treatment with brimonidine, we advise starting with a small pea-sized drop to cover the entire face. Each day the size of the drop may be increased slightly until the desired clinical result is achieved. We also do not initiate use of brimonidine in the same month as initiating use with a topical retinoid or retinol since these can all affect the already disrupted cutaneous barrier in a patient suffering with rosacea.

\section{CONCLUSION}

Rosacea is a common chronic inflammatory condition of the facial skin with a spectrum of clinical features that can wax and wane over time. Many of these clinical features overlap in the four recognized clinical subtypes of the disease $[1,2]$. Persistent facial erythema is a very common and difficult to treat manifestation of many cases of rosacea, and until recently represented a significant gap in the therapeutic management of the disease. Flushing is a distinct symptom of rosacea that involves rapid-onset superficial cutaneous vasodilatation in response to emotional triggers, whereas transient or persistent erythema may be a result of the multifactorial inflammatory process promoting vasodilatation and neovascularization. Brimonidine tartrate topical gel is the first approved agent for the treatment of facial erythema associated with rosacea and acts through vasoconstriction of small caliber subcutaneous blood vessels via specific agonism of the post-synaptic $\alpha 2 \mathrm{~A}$-adrenergic receptor on endothelial cells.
Significant improvement in erythema is experienced in the majority of patients with once-daily topical application with few experiencing mild to moderate cutaneous adverse effects that are often not persistent with continued use [34]. This provides clinicians with a safe and effective treatment modality for this previously difficult to treat manifestation of rosacea, and it has been shown to be safely used in combination with other therapeutics targeting the inflammatory lesions of the disease. Currently this is a short-term symptomatic treatment with significant beneficial effects that wear off by around $12 \mathrm{~h}$ after application. Further studies are needed to address the hypothetical question that continued long-term use of this medication may permanently alter neurovascular mechanisms behind persistent erythema in rosacea and possibly alter the course of this clinical manifestation over time.

\section{ACKNOWLEDGMENTS}

No funding or sponsorship was received for this study or publication of this article. All named authors meet the International Committee of Medical Journal Editors (ICMJE) criteria for authorship for this manuscript, take responsibility for the integrity of the work as a whole, and have given final approval for the version to be published. We thank Alison Harvey and Anna Holmes of Galderma for ensuring the scientific accuracy of the manuscript. While they helped with providing some of the references, they did not control the content or the conclusions of the article. During the peer review process, the manufacturer of the agent under review was offered an opportunity to comment on the article. Changes resulting from comments received were made by the 
author based on their scientific and editorial merit.

Conflict of interest. A. W. Johnson has no conflicts of interest. S. M. Johnson is a speaker, investigator and advisor for Galderma; an investigator and advisor for Nielsen; an investigator for Regeneron; a speaker for Sun Pharmaceuticals; investigator for AbbVie; and an investigator and advisor for Johnson \& Johnson.

Compliance with ethics guidelines. This article is based on previously conducted studies and does not involve any new studies of human or animal subjects performed by any of the authors.

Open Access. This article is distributed under the terms of the Creative Commons Attribution Noncommercial License which permits any noncommercial use, distribution, and reproduction in any medium, provided the original author(s) and the source are credited.

\section{REFERENCES}

1. Leyden JJ. The evolving view of rosacea. J Am Acad Dermatol. 2013;69:S1.

2. Elewski BE, Draelos Z, Dreno B, et al. Rosaceaglobal diversity and optimized outcome: proposed international consensus from the Rosacea International Expert Group. J Eur Acad Dermatol Venereol. 2011;25:188-200.

3. Del Rosso JQ, Thiboutot D, Gallo R, et al. Consensus recommendations from the American Acne \& Rosacea Society on the management of rosacea, part 2: a status report on topical agents. Cutis. 2013;92:277-84.

4. Wilkin J, Dahl M, Detmar M, et al. Standard classification of rosacea: report of the National Rosacea Society Expert Committee on the classification and staging of rosacea. J Am Acad Dermatol. 2002;46:584-7.
5. Tan J, Blume-Peytavi U, Ortonne JP, et al. An observational cross-sectional survey of rosacea: clinical associations and progression between subtypes. Br J Dermatol. 2013;169:555-62.

6. Del Rosso JQ, Gallo RL, Kircik L, et al. Why is rosacea considered to be an inflammatory disorder? The primary role, clinical relevance, and therapeutic correlations of abnormal innate immune response in rosacea-prone skin. J Drugs Dermatol. 2012;11:694-700.

7. Steinhoff M, Schauber J, Leyden JJ. New insights into rosacea pathophysiology: a review of recent findings. J Am Acad Dermatol. 2013;69:S15-26.

8. Del Rosso JQ, Gallo RL, Tanghetti E, Webster G, Thiboutot D. An evaluation of potential correlations between pathophysiologic mechanisms, clinical manifestations, and management of rosacea. Cutis. 2013;91:1-8.

9. Del Rosso JQ. Advances in understanding and managing rosacea: part 1: connecting the dots between pathophysiological mechanisms and common clinical features of rosacea with emphasis on vascular changes and facial erythema. J Clin Aesthet Dermatol. 2012;5:16-25.

10. Del Rosso JQ. Advances in understanding and managing rosacea: part 2: the central role, evaluation, and medical management of diffuse and persistent facial erythema of rosacea. J Clin Aesthet Dermatol. 2012;5:26-36.

11. Parodi A, Drago F, Paolino S, Cozzani E, Gallo R. Treatment of rosacea. Ann Dermatol Venereol. 2011;138(Suppl 3):S211-4.

12. Feldman SR, Huang WW, Huynh TT. Current drug therapies for rosacea: a chronic vascular and inflammatory skin disease. J Manag Care Spec Pharm. 2014;20:623-9.

13. McClellan KJ, Noble S. Topical metronidazole. A review of its use in rosacea. Am J Clin Dermatol. 2000;1:191-9.

14. Leeming JP, Holland KT, Bojar RA. The in vitro antimicrobial effect of azelaic acid. Br J Dermatol. 1986;115:551-6.

15. Gupta AK, Nicol K. The use of sulfur in dermatology. J Drugs Dermatol. 2004;3:427-31.

16. Del Rosso JQ. Evaluating the role of topical therapies in the management of rosacea: focus on combination sodium sulfacetamide and sulfur formulations. Cutis. 2004;73:29-33. 
17. Abokwidir M, Fleischer AB. An emerging treatment: topical ivermectin for papulopustular rosacea. J Dermatolog Treat. 2015. doi:10.3109/09546634. 2014.991672.

18. Del Rosso JQ. Management of facial erythema of rosacea: what is the role of topical alpha-adrenergic receptor agonist therapy? J Am Acad Dermatol. 2013;69:S44-56.

19. Fowler J Jr, Jackson M, Moore A, et al. Efficacy and safety of once-daily topical brimonidine tartrate gel $0.5 \%$ for the treatment of moderate to severe facial erythema of rosacea: results of two randomized, double-blind, and vehicle-controlled pivotal studies. J Drugs Dermatol. 2013;12:650-6.

20. Shanler SD, Ondo AL. Successful treatment of the erythema and flushing of rosacea using a topically applied selective alpha1-adrenergic receptor agonist, oxymetazoline. Arch Dermatol. 2007;143:1369-71.

21. Piwnica D, Rosignoli C, de Menonville ST, et al. Vasoconstriction and anti-inflammatory properties of the selective alpha-adrenergic receptor agonist brimonidine. J Dermatol Sci. 2014;75:49-54.

22. Benkali K, Leoni M, Rony F, et al. Comparative pharmacokinetics and bioavailability of brimonidine following ocular and dermal administration of brimonidine tartrate ophthalmic solution and gel in patients with moderate-tosevere facial erythema associated with rosacea. Br J Dermatol. 2014;171:162-9.

23. Fowler J, Jarratt M, Moore A, et al. Once-daily topical brimonidine tartrate gel $0.5 \%$ is a novel treatment for moderate to severe facial erythema of rosacea: results of two multicentre, randomized and vehicle-controlled studies. $\mathrm{Br} \mathrm{J}$ Dermatol. 2012;166:633-41.

24. Moore A, Kempers S, Murakawa G, et al. Long-term safety and efficacy of once-daily topical brimonidine tartrate gel $0.5 \%$ for the treatment of moderate to severe facial erythema of rosacea: results of a 1-year open-label study. J Drugs Dermatol. 2014;13:56-61.

25. Ilkovitch D, Pomerantz RG. Brimonidine effective but may lead to significant rebound erythema. J Am Acad Dermatol. 2014;70:e109-10.
26. Routt ET, Levitt JO. Rebound erythema and burning sensation from a new topical brimonidine tartrate gel 0.33\%. J Am Acad Dermatol. 2014;70:e37-8.

27. Tanghetti EA, Jackson JM, Belasco KT, et al. Optimizing the use of topical brimonidine in rosacea management: panel recommendations. J Drugs Dermatol. 2015;14:33-40.

28. Serle JB. A comparison of the safety and efficacy of twice daily brimonidine $0.2 \%$ versus betaxolol $0.25 \%$ in subjects with elevated intraocular pressure. The Brimonidine Study Group III. Surv Ophthalmol. 1996;41(Suppl 1):39-47.

29. Katz LJ. Brimonidine tartrate $0.2 \%$ twice daily vs timolol $0.5 \%$ twice daily: 1-year results in glaucoma patients. Brimonidine Study Group. Am J Ophthalmol. 1999;127:20-6.

30. Del Rosso JQ, Thiboutot D, Gallo R, et al. Consensus recommendations from the American Acne \& Rosacea Society on the management of rosacea, part 5: a guide on the management of rosacea. Cutis. 2014;93:134-8.

31. Del Rosso JQ, Thiboutot D, Gallo R, et al. Consensus recommendations from the American Acne \& Rosacea Society on the management of rosacea, part 1: a status report on the disease state, general measures, and adjunctive skin care. Cutis. 2013;92:234-40.

32. Galderma Laboratories LP. Mirvaso (brimonidine) $0.33 \%$ topical gel product information. 2013. www. accessdata.fda.gov/drugsatfda_docs/label/2013/ 204708lbl.pdf. Accessed 22 Apr 2014.

33. Swanson LA, Warshaw EM. Allergic contact dermatitis to topical brimonidine tartrate gel $0.33 \%$ for treatment of rosacea. JAAD. 2014;71(4):832-3.

34. Layton AM, Schaller M, Homey B, Hofmann M, Bewley A, Lehmann P, et al. Brimonidine $3 \mathrm{mg} / \mathrm{g}$ gel improves patient-reported outcomes in severe facial erythema of rosacea. In: EADV. 2014; P1853. 